\title{
Blind Timing and Carrier Synchronization in Decode and Forward Cooperative Systems
}

\author{
Ali A. Nasir, Salman Durrani and Rodney A. Kennedy \\ School of Engineering, CECS, The Australian National University, Canberra, Australia. \\ Email: \{ali.nasir, salman.durrani, rodney.kennedy\}@ anu.edu.au
}

\begin{abstract}
Synchronization in Decode and Forward (DF) cooperative communication systems is a complex and challenging task requiring estimation of many independent timing and carrier offsets at each relay in the broadcasting phase and multiple timing and carrier offsets at the destination in the relaying phase. This paper presents a scheme for blind channel, timing and carrier offset estimation in a DF cooperative system with one source, $M$ relays and one destination equipped with $N$ antennas. In particular, we exploit blind source separation at the destination to convert the difficult problem of jointly estimating multiple synchronization parameters in the relaying phase into more tractable sub-problems of estimating many individual timing and carrier offsets for the independent relays. We also modify and propose a criterion for best relay selection at the destination. Simulation results demonstrate the excellent end-toend Bit Error Rate (BER) performance of the proposed blind scheme with relay selection, which is shown to achieve the maximum diversity order with $M=4$ relays using $N=5$ antennas at the destination. The presented work is a complete solution to blind synchronization and channel estimation in DF cooperative communication systems.
\end{abstract}

\section{INTRODUCTION}

Recent studies have demonstrated that suitable cooperation among distributed users can provide the well known benefits of centralized Multiple Input Multiple Output (MIMO) systems in cooperative communication systems [1]. The basic principle of cooperation is that multiple nodes create a virtual multipleantenna system by sharing antennas of neighboring users. However, a majority of the work in the area of cooperative communications assumes perfect synchronization. It has been shown that if the synchronization errors are large, the performance is hugely degraded and the benefits of cooperation may even vanish [2], [3]. The presence of multiple distributed nodes, each with its own local oscillator, gives rise to multiple timing and multiple frequency offsets in cooperative systems. Thus estimating multiple synchronization parameters is a challenging task that is key to future deployment of cooperative communication systems.

Prior work in this area has looked at estimating either multiple timing offsets or multiple frequency offsets using a non-blind approach. Multiple carrier offset estimation using pilots or training sequences has been considered in the context of general MIMO systems [4], MIMO-OFDM systems [5][7], sensor networks [8] and Decode and Forward (DF) and Amplify and Forward (AF) cooperative networks [9]. Multiple timing offset estimation has been considered in the context of DF cooperative systems [10], using pilots to design a resynchronization filter to align asynchronous signals at the destination. In [11], a training block having tile structure in the frequency domain is proposed to estimate multiple timing and multiple frequency offsets in OFDM-based DF networks. The use of training sequences, however, reduces the data rate and may become unrealistic or impractical, especially in the context of military and defence applications and emerging wireless ad hoc networks which operate on opportunistic communication paradigm. Hence the need for blind solutions is well motivated in these contexts.

Compared to non-blind solutions, only a few authors have proposed blind solutions. A blind method of multiple carrier frequency offset estimation is proposed in [12] based on formulating a virtual MIMO system using polyphase components of the oversampled signal. In [13], [14], the approach is shown to be able to separate two users in a distributed Multiple Input Single Output (MISO) system. However for more than two users, the oversampling requirements become prohibitive and the approach becomes impractical. Recently in [15], the authors have proposed a solution to the problem of blind timing and carrier synchronization in a distributed $M$ user $\times N$ antenna system, where Blind Source Separation (BSS) is used to decouple the timing and carrier offsets from user to user and reduce the computational complexity.

In this paper, we focus on blind synchronization and channel estimation in DF cooperative communication systems with one source, $M$ relays and one destination equipped with $N$ multiple antennas. For the broadcasting phase, the synchronization problem is identical to the Single Input Single Output (SISO) and we choose the blind carrier estimation [16], timing estimation [17] and channel estimation [18] techniques respectively available in the literature. For the relaying phase, we modify and extend the BSS approach in [15] with the following important differences. First we propose a modification to the relay transmitters involving block interleaving to allow blind source separation to work in the DF cooperative system. Secondly, we exploit blind source separation at the destination to convert the difficult problem of jointly estimating multiple synchronization parameters in the relaying phase into more tractable sub-problems of estimating many individual timing and carrier offsets for the independent relay. This allows timing and carrier offsets to be estimated using the same algorithms as in the broadcasting phase. Thirdly, we propose a modification of the relay selection algorithm in [19] for the case of multiple antennas at the destination. Simulation results demonstrate 


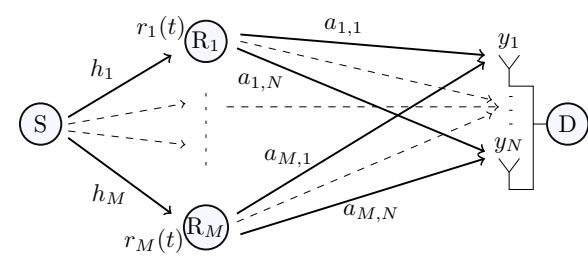

Fig. 1. System model for blind cooperative communication.

excellent end-to-end Bit Error Rate (BER) performance of the proposed blind scheme in block Rayleigh flat-fading channels and show that it is able to achieve the maximum diversity order with $M=4$ relays using $N=5$ antennas at the destination. To the best of authors knowledge, the presented work is a first complete solution in the literature to demonstrate blind synchronization and channel estimation in DF cooperative communication systems.

The rest of the paper is organised as follows. Section II describes the system model. The proposed blind synchronization scheme is discussed in Section III. The relay selection algorithm is presented in Section IV. Simulation results are discussed in Section V. Finally conclusions are drawn in Section VI.

\section{SySTEM MODEL}

We consider a two hop decode-and-forward cooperative communication system with one source node, one destination node and $M$ relay nodes. All nodes operate in half-duplex mode. The source and relay nodes are equipped with a single antenna while the destination node is equipped with an array of $N$ antenna elements as shown in Fig. 1. The flat-fading coefficients for the source to relay and relay to destination links are denoted by $h_{m}$ and $a_{m, j}$ respectively, where $m=1,2, \ldots, M$ is the relay index and $j=1,2, \ldots, N$ is the antenna index. These coefficients are modeled as zeromean complex Gaussian random variables with unit variance, leading to a Rayleigh fading channel model. In addition, we assume quasi-static fading coefficients, i.e., all the fading coefficients are constant for a frame of transmitted bits but are independent and identically (i.i.d.) distributed from frame to frame.

Communication between the source node and destination node takes place in two phases. In the broadcasting phase, the source node broadcasts a frame of $D$ modulated symbols to relay nodes. Each relay node operates independently and blindly estimates its channel, timing and carrier offsets before decoding the incoming information. In the relaying phase, each relay interleaves and then transmits the decoded data to the destination. The multiple antennas at the destination node help to achieve Blind Source Separation (BSS) of multiple relay signals (cf. Section III-B). After BSS, the best relay is selected (cf. Section IV) and its timing and carrier offsets are blindly estimated. Finally the synchronized best relay signal is de-interleaved and the transmitted information is decoded. Note that training sequences are not required for synchronization in any of the communication phases.

\section{BLIND TIMING AND CARRIER SYNCHRONIZATION}

The proposed scheme for blind synchronization in the broadcasting phase and the relaying phase of the DF cooperative communication system is presented in this section.

\section{A. Synchronization in Broadcasting Phase}

The synchronization problem in the broadcasting phase is to estimate the channel coefficient $h_{m}$, timing offset $\tau_{s, m}$ and carrier offset $f_{s, m}$ at each relay without pilots or training sequences, where subscript $s$ denotes the source and $m$ is the relay index. Note that since all the relays act independently, the synchronization problem in the broadcasting phase is the same as in Single Input Single Output (SISO) systems, for which blind solutions already exist in the literature. See, for example, [12], [18], [20] and references therein.

Let $x(t)$ be the pulse modulated baseband signal which is transmitted by the source and is given by

$$
x(t)=\sum_{k=0}^{D-1} d(k) g_{T}(t-k T),
$$

where $d(k)$ denotes the complex valued modulation symbol $k$ taken from an $M$-ary PSK (Phase Shift Keying) constellation, $g_{T}(t)$ is the transmitter pulse shape, $T$ is the symbol period and $D$ is the transmission block length. The signal is up converted to the carrier frequency $\omega_{s}$, as shown in the Fig. 2, before transmission.

At each relay, the received signal $r_{m}(t)$ is down converted by the oscillator frequency, $-\omega_{s}+\omega_{s, m}$, where $\omega_{s, m}$ is the analog frequency offset between the source and relay $m$. The baseband received signal at relay $m$ is given by

$\tilde{r}_{m, \tau_{m}}(t)=h_{m} \sum_{k=0}^{D-1} d(k) g_{T}\left(t-k T-\tau_{s, m} T\right) e^{j \omega_{s, m} t}+v_{r, m}(t)$

where ' $\tilde{~ ' ~ o v e r ~} r_{m, \tau_{m}}(t)$ indicates the received signal with frequency offset corruption, $h_{m}$ is the complex channel coefficient from source to relay $m, v_{r, m}(t)=n_{r, m}(t) e^{-j\left(\omega_{s}-\omega_{s, m}\right) t}$ is the noise term for relay $m, n_{r, m}(t)$ is the zero mean additive white Gaussian noise (AWGN), $\tau_{s, m}$, normalized by the symbol duration $T$, is the fractional unknown timing offset, $\left|\tau_{s, m}\right| \leq 1 / 2$, between the source and relay $m$.

After sampling, the received signal at each relay can be written as

$$
\begin{aligned}
\tilde{r}_{m, \tau_{m}}\left(b T_{s}\right)=h_{m} \sum_{k=0}^{D-1} d(k) g_{T}\left(b T_{s}-k T\right. & \left.-\tau_{s, m} T\right) e^{j 2 \pi f_{s, m} b} \\
& +v_{r, m}\left(b T_{s}\right)
\end{aligned}
$$

where $b$ is the sampling index, $\omega_{s, m} t=\omega_{s, m} b T_{s}=$ $2 \pi\left(F_{s, m} / F_{s}\right) b, F_{s, m}$ is the frequency offset in $\mathrm{Hz}$ and $f_{s, m}=$ $F_{s, m} / F_{s}$ is the digital frequency offset in cycles/sample between source and relay $m$. Thus, the blind timing and carrier synchronization problem in the broadcasting phase can be expressed as determining $\tau_{s, m}$ and $f_{s, m}$ at each relay, given the received signal, $\tilde{r}_{m, \tau_{m}}(t)$ at relay. 


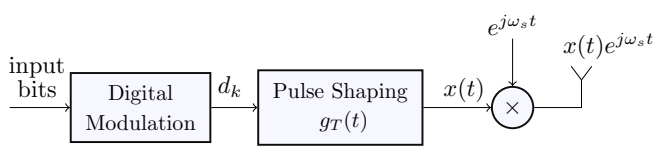

Fig. 2. Source Transmitter.

Proposed Relay Receiver: The block diagram of the proposed blind relay receiver is shown in Fig. 3. We propose to do carrier offset estimation first since carrier offset is a very sensitive parameter and must be estimated very accurately. We choose to estimate the frequency offset using non-linear least square approach by exploiting the fourth-order conjugate cyclostationary statistics of the received signal [16] (see Appendix A for estimator summary). The frequency offset correction is performed as shown in Fig. 3 with output

$$
\begin{array}{r}
\hat{\mathbf{x}}_{h_{m}, \tau_{s, m}}\left(b T_{s}\right)=h_{m} \sum_{k=0}^{D-1} d(k) g_{T}\left(b T_{s}-k T-\tau_{s, m} T\right)+ \\
\hat{v}_{r, m}\left(b T_{s}\right)
\end{array}
$$

which is an estimate of baseband source output signal, $x(t)$, corrupted by timing offset and the channel, $\hat{v}_{r, m}\left(b T_{s}\right)=$ $v_{r, m}\left(b T_{s}\right) e^{-j 2 \pi \hat{f}_{s, m} b}$ and $\hat{f}_{s, m}$ is an estimate of frequency offset between source and relay $m$.

The next step is to estimate the timing offset, for which any blind SISO timing estimation algorithm can be used. In this work, we choose the blind feedforward timing offset estimator using Square-Law Nonlinearity [17] (see Appendix B for estimator summary). The timing offset estimate $\hat{\tau}_{s, m}$ is then used by the matched filter and the signal $\hat{\mathbf{x}}_{h_{m}, \tau_{s, m}}$ is filtered with impulse response $g_{R}\left(t+\hat{\tau}_{s, m}\right)$, which is matched to the transmit filter $g_{T}(t)$. Matched filter output, $\hat{\mathbf{x}}_{h_{m}}$, is given as

$$
\hat{\mathbf{x}}_{h_{m}}\left(b T_{s}\right)=h_{m} \sum_{k=0}^{D-1} d(k) g_{T}\left(b T_{s}-k T\right)+\hat{v}_{r, m}^{\prime}\left(b T_{s}\right)
$$

where the sequence $\hat{\mathbf{x}}_{h_{m}}$ at the symbol rate is an estimate of $x(t)$ free from offsets but containing the effect of the channel.

Finally, we estimate the channel using cumulant interference subspace cancelation method [18] (see Appendix C for estimator summary). Equalization is performed on the basis of channel estimate and demodulator generates the decoded bits, as illustrated in Fig. 3. Note that the blind channel estimation is performed last since the algorithm assumes perfect timing and carrier synchronization for its proper operation.

\section{B. Synchronization in Relaying Phase}

The synchronization problem in the relaying phase is more difficult and complicated than in the broadcasting phase. This is because of the need to estimate multiple timing and carrier offsets at the destination node. In this work, we follow the general approach in [15] and use Blind Source Separation (BSS) at the destination node to decouple the timing and carrier offsets from user to user. This converts the difficult problem of estimating multiple offsets into more tractable sub-problem of estimating many independent timing and carrier offsets. For BSS, we use the JADE (Joint Approximate Diagonalization of

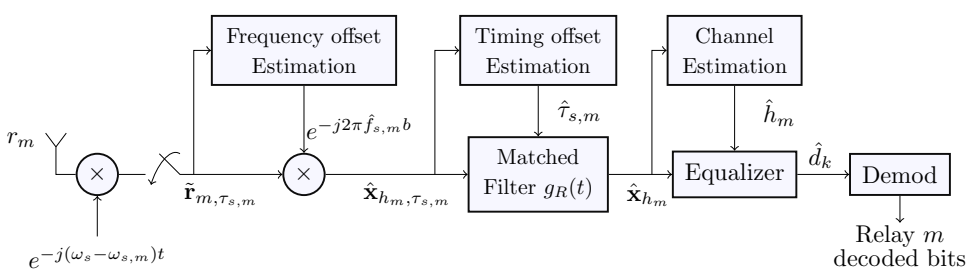

Fig. 3. Relay $m$ receiver.

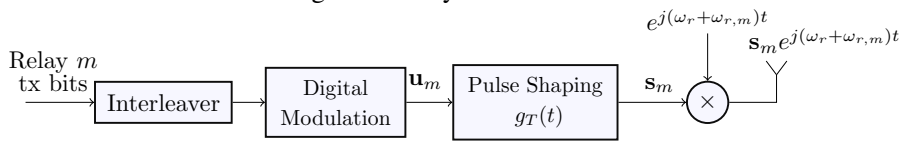

Fig. 4. Relay $m$ transmitter.

Eigen matrices) algorithm [21] (see Appendix D for summary of JADE algorithm). $N \geq M$ antennas at the destination are necessary to provide the diversity for proper working of JADE. Since JADE requires input signals to be statistically independent, we propose that each relay independently interleaves the decoded data before transmission.

Proposed Relay Transmitter: The block diagram of the proposed relay $m$ transmitter is shown in Fig. 4. Each relay $m$ interleaves the decoded bits using a matrix interleaver with $m$ rows and $(D \times K) / m$ columns, where $K$ is the number of bits per symbol, $m$ is the relay index and $D$ is the frame length. After digital modulation and pulse shaping, the transmitted baseband signal of relay $m$ is given as

$$
s_{m}(t)=\sum_{k=0}^{D-1} u_{m}(k) g_{T}(t-k T), \quad \text { for } \quad m=1, \ldots, M
$$

where $u_{m}(k)$ denotes the complex valued data symbol $k$ of relay $m$. The signal is up converted to the carrier frequency $\omega_{r}+\omega_{r, m}$, where $\omega_{r}$ is the demodulator carrier frequency at the destination and $\omega_{r, m}$ is the analog frequency offset between the relay $m$ and the destination.

Proposed Destination Receiver: The block diagram of the proposed destination receiver is shown in Fig. 5. The frequency demodulators associated with all the antennas are fed by the same oscillator with frequency $\omega_{r}$. Moreover, it is assumed that all the samplers at each destination antenna operate at the same time instant. The sampled signal at antenna $i$ is given as

$$
\begin{array}{r}
\tilde{y}_{i, \tau}\left(b T_{s}\right)=\sum_{m=1}^{M} a_{m, i} \sum_{k=0}^{D-1} u_{m}(k) g_{T}\left(b T_{s}-k T-\tau_{r, m} T\right) \times \\
e^{j 2 \pi f_{r, m} b}+v_{d, i}\left(b T_{s}\right)
\end{array}
$$

where $b$ is the sampling index, $f_{r, m}$ is the digital frequency offset and $\tau_{r, m}$ is the timing offset between relay $m$ and the destination, $a_{m, i}$ is the channel coefficient between relay $m$ and antenna $i$ and $v_{d, i}(t)$ is the noise term at antenna $i$.

After BSS, the separated relay signals can be written as

$$
\begin{array}{r}
\hat{\tilde{s}}_{m, \tau_{r, m}}\left(b T_{s}\right)=\sum_{k=0}^{D-1} u_{m}(k) g_{T}\left(b T_{s}-k T-\tau_{r, m} T\right) e^{j 2 \pi f_{r, m} b}+ \\
\breve{v}_{d, m}\left(b T_{s}\right)
\end{array}
$$




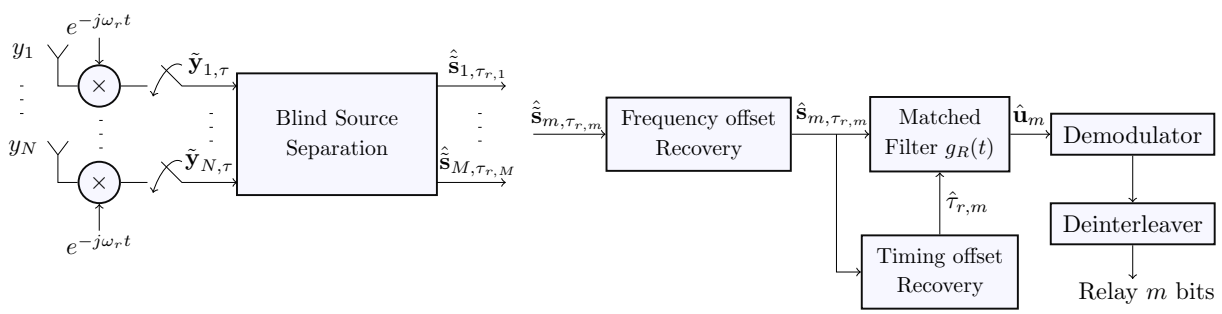

Fig. 5. Destination Receiver.

where $\hat{\tilde{s}}_{m, \tau_{r, m}}\left(b T_{s}\right)$ is the baseband transmitted signal of relay $m$ corrupted by timing and frequency offset and $\breve{v}_{d, m}\left(b T_{s}\right)$ is the noise element after BSS.

We assume that the destination selects the best relay after blind source separation. For carrier offset and timing offset estimation on the selected relay signal, we use the same algorithms as in the broadcasting phase. Finally, frequency and timing offset recovery yields an estimate of interleaved data symbols at the symbol rate, $\hat{u}_{m}(k)$, for the selected relay $m$ from which the transmitted data can be decoded. The relay selection procedure is discussed in the next section.

\section{Relay Selection Algorithm}

For DF relaying, various decoder strategies have been proposed in the literature that have been shown to achieve the full diversity order $M$, where $M$ is the number of relays, in terms of the Bit Error Rate (BER) performance [22][24]. In this regard, relay selection has emerged as a simple, powerful technique with comparatively lower implementation complexity. Various criteria for "best" relay section in relaying phase have been proposed in the literature assuming destination has single antenna. Recently, a method is proposed in [19] in which the destination chooses the best relay based on the minimum of the source-to-relay and relay-to-destination Signal to Noise Ratios. The implementation of this scheme requires Channel State Information (CSI) of source to relay channels at the destination, which can be easily done in practice through a feedforward channel from the relay to the destination [19]. It is shown that the algorithm has superior performance compared to existing methods and is able to extract the full diversity without requiring any feedback CSI or error detection mechanism such as cyclic redundancy check at relay nodes.

In our work, we modify the criterion in [19] for the case where destination has multiple antennas. In our scheme, after blind source separation, the destination chooses the best relay based on the following criterion

$$
R_{\text {sel }}=\arg \max _{m}\left\{\min \left\{h_{m}, \min _{j}\left\{a_{m j}\right\}\right\}\right\}
$$

where "sel" denotes the selected relay $m$. Note that the relay to destination channels $a_{m j}$ are blindly estimated at the destination using JADE, while information about estimated source to relay channels $h_{m}$ is assumed to be available at the destination via a feedforward channel from the relay to the destination. For comparison, we also consider the case of "no relay selection" in which the destination randomly selects one of the relays.

The carrier offset and timing offset estimation is then carried for the selected relay and the data is demodulated and deinterleaved as shown in Fig. 5. The performance of the proposed blind DF cooperative communication system with and without relay selection is illustrated in the next section.

\section{Simulation Results}

In this section, simulation results are presented to validate the robustness of the proposed blind cooperative communication system. We consider $M=4$ relays and a destination equipped with $N=5$ antennas ${ }^{1}$. Each transmission from the source or a relay employs Quadrature Phase Shift Keying (QPSK) modulation and transmits data in frames of length $D=400$ symbols. The propagation channels are i.i.d. block Rayleigh flat-fading channels. The root raised cosine filters are used for transmitter pulse shaping and receiver matched filtering, with roll-off factor, $\beta=0.25$ and filter tap delay length set to 5 symbols. The oversampling factor used is $Q=4$. The unknown timing offsets are assumed to be uniformly distributed as $|\tau| \leq 1 / 2$. The unknown digital frequency offsets are assumed to be uniformly distributed as $|f| \leq 1 / 32$. The timing offset estimation parameter $L=16$ (see Appendix B). The figures of merit used are the end-toend (i.e., source to destination) Bit Error Rate (BER) and the Mean Square Error (MSE) of frequency and timing offsets, respectively.

\section{A. MSE of frequency and timing offsets}

Figs. 6 and 7 show the MSE performance of frequency offset and timing offset estimation, respectively. The results are shown for both the broadcasting and relaying phase and are averaged out over the $M=4$ relays. It can be seen from the two figures that the MSE improves with increasing Signal to Noise Ratio (SNR). Compared to the broadcasting phase, the MSE is better for the relaying phase due to the use of multiple antennas at the destination. The error floor at high SNR in the MSE of frequency offset for relaying phase is due to using a finite step size in the frequency offset estimation algorithm (see Appendix A).

\footnotetext{
${ }^{1}$ For $M$ user, $N=M+1$ antennas provides close to optimum blind source separation performance [15].
} 


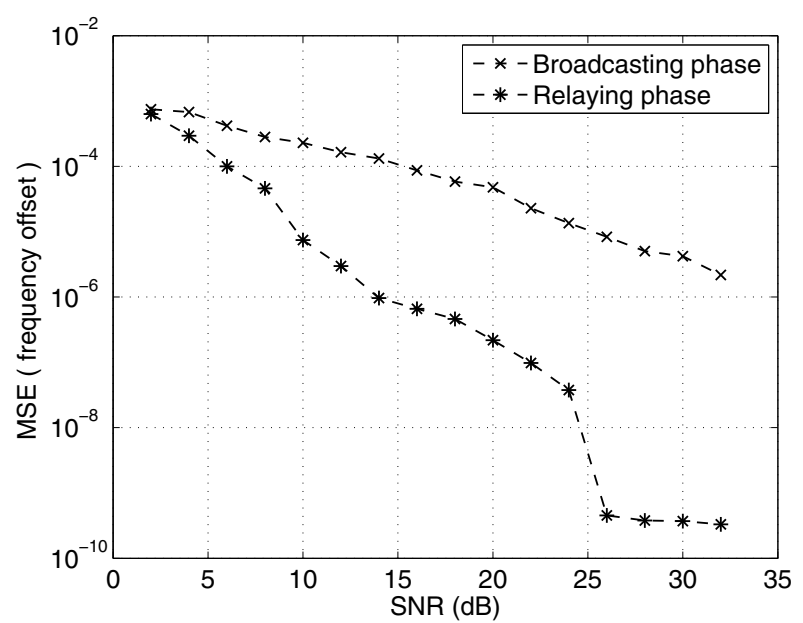

Fig. 6. MSE of frequency offset estimation as a function of SNR (dB), with $M=4$ Relays and $N=5$ antennas at destination.

\section{B. End-to-End BER}

Fig. 8 shows the end-to-end (i.e., source to destination) BER of blind DF cooperative communication as a function of SNR. The following systems are considered for comparison:

1) A blind cooperative DF system operating in absence of timing and carrier offsets and endowed with perfect BSS and employing relay selection, since its performance represents a lower bound (labelled by "Lower bound").

2) A blind cooperative DF system operating in absence of timing and carrier offsets but with BSS and employing relay selection, since its performance allows effect of BSS to be studied (labelled "BSS with no offsets").

3) Proposed cooperative DF system estimating channel, timing and carrier offsets with BSS and relay selection (labelled by "Proposed - with relay selection").

4) Proposed cooperative DF system estimating channel, timing and carrier offsets with BSS but no relay selection (labelled by "Proposed - no relay selection").

It can be seen that the slope of the "Proposed - no relay selection" BER result achieves the theoretical diversity order of 1 , which confirms the correct working of the blind synchronization and channel estimation algorithms in the proposed scheme. The other three BER results which use relay selection, including the "Proposed - no relay selection" (which is the actual system performance), all have the same slope and achieve the theoretical diversity order of 4 for medium to high SNR, without any noticeable error floor in the considered SNR range. This confirms the correct working of the proposed relay selection algorithm. The "BSS with no offsets" and "Proposed - with relay selection" curves are very close to each other, indicating that the performance loss due to synchronization errors is very small. Finally, comparing the "Lower bound" and the "BSS with no offsets" curves, we can see that they have the same slope and the gap between them is due to self noise of the JADE algorithm. Similar results have also been found for 2, 3 and 5 relays, but are not shown here due to space limitations.

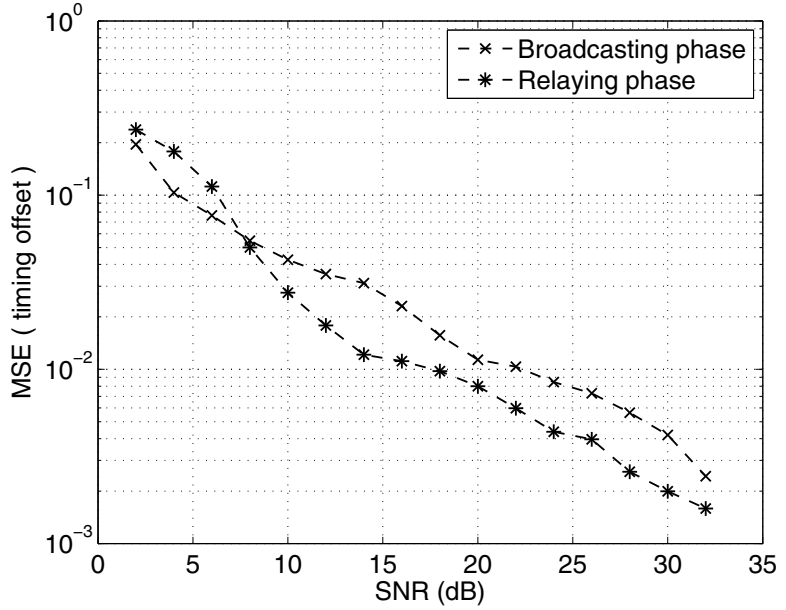

Fig. 7. MSE of timing offset estimation as a function of SNR (dB), with $M=4$ Relays and $N=5$ antennas at destination.

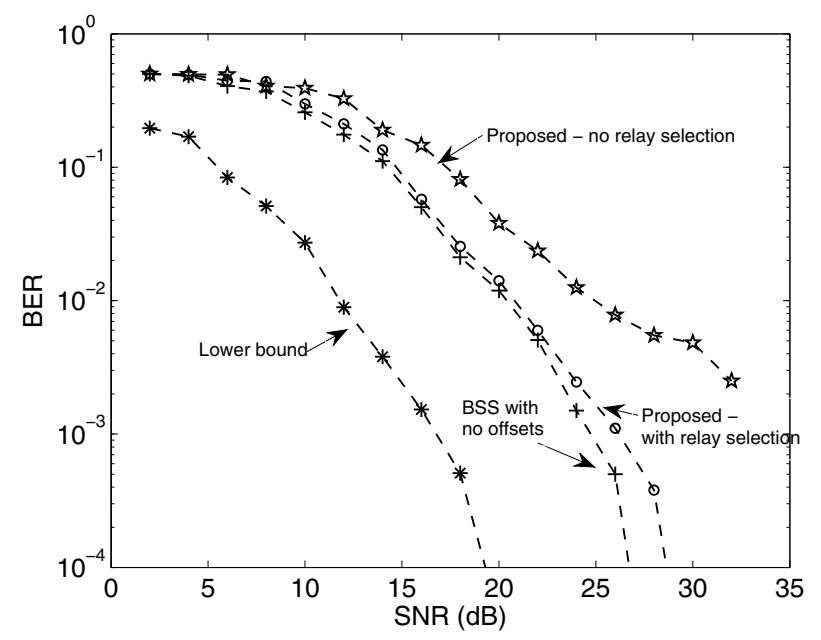

Fig. 8. End-to-end BER of blind DF cooperative communication as a function of SNR (dB), with $M=4$ Relays and $N=5$ antennas at destination.

\section{CONCLUSIONS}

In this paper, we have proposed a scheme for blind synchronization and channel estimation in DF cooperative communication systems. Blind source separation using JADE algorithm has been proposed to decouple the timing and carrier offsets from user to user in the relaying phase. This allows timing and carrier offsets to be estimated using blind SISO square-law and cyclostationary algorithms respectively in both the broadcasting and relaying phases. The channels in the broadcasting phase are estimated using blind SISO cumulant interference subspace cancellation algorithm. In addition, a relay selection algorithm has been proposed to select the best relay for decoding source information at the destination. Results have demonstrated that the proposed blind scheme works satisfactorily in terms of end-to-end BER and is able to extract the maximum diversity order due to $M$ relays with $N=M+1$ antennas at the destination. 


\section{REFERENCES}

[1] A. Scaglione, D. L. Goeckel, and J. N. Laneman, "Cooperative communications in mobile ad hoc networks," IEEE Signal Processing Magazine, vol. 23, no. 5, pp. 18-29, Sept. 2006.

[2] P. A. Parker, P. Mitran, D. W. Bliss, and V. Tarokh, "On bounds and algorithms for frequency synchronization for collaborative communication systems," IEEE Trans. Signal Process., vol. 56, pp. 3742-3752, 2008.

[3] A. S. Ibrahim and R. Liu, "Mitigating channel estimation error with timing synchronization tradeoff in cooperative communications," IEEE Trans. Signal Process., vol. 58, pp. 337-348, 2010.

[4] O. Besson and P. Stoica, "On parameter estimation of MIMO flat-fading channels with frequency offsets," IEEE Trans. Signal Process., vol. 51, no. 3, pp. 602-613, Mar. 2003.

[5] Y. Zhang and J. Zhang, "Mulitple CFOs compensation and BER analysis for cooperative communication systems," in Proc. IEEE WCNC, 2009.

[6] J. Zhang, Y. R. Zheng, C. Xiao, and K. B. Letaief, "Channel equalization and symbol detection for single-carrier MIMO systems in the presence of multiple carrier frequency offsets," IEEE Trans. Veh. Technol., vol. 59, no. 4, pp. 2021-2030, may 2010.

[7] L. Haring, S. Bieder, A. Czylwik, and T. Kaiser, "Estimation algorithms of multiple channels and carrier frequency offsets in application to multiuser OFDM systems," IEEE Trans. Wireless Commun., vol. 9, no. 3, pp. 865-870, Mar. 2010.

[8] M. Oh, X. Ma, G. Giannakis, and D. Park, "Cooperative synchronization and channel estimation in wireless sensor networks," Journal on Communications and Networks, vol. 7, no. 3, pp. 284-293, Sep. 2005.

[9] H. Mehrpouyan and S. Blostein, "Synchronization in cooperative networks: Estimation of multiple carrier frequency offsets," in Proc. IEEE ICC, 2010.

[10] X. Li, Y. C. Wu, and E. Serpedin, "Timing synchronization in decodeand-forward cooperative communication systems," IEEE Trans. Signal Process., vol. 57, no. 4, pp. 1444-1455, Apr. 2009.

[11] Q. Huang, M. Ghogho, J. Wei, and P. Ciblat, "Practical timing and frequency synchronization for OFDM based cooperative systems," IEEE Trans. Signal Process., vol. 58, no. 7, pp. 3706-3716, Jul. 2010.

[12] Y. Yu, A. Petropulu, H. Poor, and V. Koivunen, "Blind estimation of multiple carrier frequency offsets," in Proc. IEEE PIMRC, 2007.

[13] X. Liu, A. Petropulu, H. Poor, and V. Koivunen, "Blind separation of two users based on user delays and optimal pulse shape design," EURASIP Journal on Wireless Communications and Networking, Apr. 2010.

[14] X. Liu, J. Kountouriotis, A. Petropulu, and K. R. Dandekar, "ALOHA with collision resolution (ALOHA-CR): Theory and software defined radio implementation," IEEE Trans. Signal Process., vol. 58, no. 8, pp. 4396-4410, Aug. 2010.

[15] A. A. Nasir, S. Durrani, and R. A. Kennedy, "Blind timing and carrier synchronization in distributed MIMO communication systems," submitted to IET Transactions on Communications, Jun. 2010.

[16] Y. Wang, K. Shi, and E. Serpedin, "Non data-aided feedforward carreir frequency offset estimators for QAM constellations: A nonlinear leastsquares approach," EURASIP Journal on Applied Signal Processing, vol. 13, pp. 1993-2001, 2004.

[17] M. Oerder and H. Meyr, "Digital filter and square timing recovery," IEEE Trans. Commun., vol. 36, no. 5, pp. 605-612, May 1988.

[18] J. Fang, A. R. Leyman, Y. Chew, and Y. C. Liang, "A cumulant interference subspace cancellation method for blind SISO channel estimation," IEEE Trans. Signal Process., vol. 54, pp. 784-790, 2006.

[19] M. Fareed and M. Uysal, "On relay selection for decode-and-forward relaying," IEEE Trans. Wireless Commun., vol. 8, no. 7, pp. 3341-3346, Jul. 2009.

[20] Y.-C. Wu and E. Serpedin, "Unified analysis of a class of blind feedforward symbol timing estimators employing second-order statistics," IEEE Trans. Wireless Commun., vol. 5, no. 4, pp. 737-742, Apr. 2006.

[21] J. F. Cardoso, "Blind signal separation: Statistical principles," Proc. IEEE, vol. 86, no. 10 , pp. 2009-2025, 1998

[22] Y. Jing and H. Jafarkhani, "Single and multiple relay selection schemes and their achievalbe diveristy orders," IEEE Trans. Wireless Commun., vol. 8, no. 3, pp. 1414-1423, Mar. 2009.

[23] Y. Fan, J. Thompson, A. Adinoyi, and H. Yanikomeroglu, "Space diversity for mulit-antenna multi-relay channels," in Proc. 12th European Wireless Conference, 2006.

[24] H. Muhaidat and M. Uysal, "Cooperative diversity with multiple antenna nodes in fading relay channels," IEEE Trans. Wireless Commun., vol. 7 , no. 8, pp. 3036-3046, Aug. 2008.

\section{APPENDIX A}

FREQUENCY OFFSET ESTIMATION

The frequency offset estimate $\hat{f}$ is given by

$$
\hat{f}=\frac{1}{Q} \arg \max _{|\dot{\alpha}|<1 /(2 Q)} J(\dot{\alpha})
$$

where $Q$ is the oversampling factor, $\dot{\alpha}$ is a trial value of the frequency and objective function $J(\cdot)$ is given by

$$
J(\dot{\alpha})=\sum_{j=0}^{Q-1}\left|\frac{1}{R} \sum_{b=0}^{R-1} s^{4}(b) e^{-j 2 \pi(\dot{\alpha}+q / Q) b}\right|^{2}
$$

where $q=0,1, \ldots, Q-1, R$ is the length of the signal $s$, which is corrupted by the carrier offset. Note that the above estimator can handle frequency offsets in the range $(|f| \leq$ $1 / 8 Q T)$ where $T$ is the symbol time.

\section{APPENDIX B}

TIMING OFFSET ESTIMATION

The timing offset estimate $\hat{\tau}$ is given by

$$
\hat{\tau}=E_{r}\left\{-\frac{1}{2 \pi} \arg \left(\sum_{\ell=r Q L}^{(r+1) L Q-1}|s(\ell)|^{2} e^{-j 2 \pi \ell / Q}\right)\right\}
$$

where $E\{\cdot\}$ denotes expectation operator and $r$ corresponds to different sections of length $Q L$ of the squared input signal $s$, which is corrupted by the timing offset. Note that the above estimator can handle timing offsets in the range $|\tau| \leq 1 / 2$.

\section{APPENDIX C \\ BLIND CHANNEL ESTIMATION}

The blind channel estimation is described in detail in [18] and has three main steps. The first step is to compute a series of fourth order cumulant matrices of the received signal. The second step is to evaluate Singular Value Decomposition of the concatenated cumulant matrix and select left singular vectors associated with the smallest singular values. The singular vectors are in the left null space of the concatenated cumulant matrix. Finally, applying these singular vectors on the cumulant matrix extracts the channel information by canceling the interference subspace of the channel convolution matrix [18].

\section{APPENDIX D \\ BLIND SOURCE SEPARATION}

Blind source separation using JADE has two main steps. The first step is pre-whitening to make the received signal components uncorrelated. The second step is to determine the unitary transformation matrix to estimate the channels and to decouple the users. The detailed procedure is explained in [15], [21]. Note that when we use JADE, the channel matrix is estimated up to a permutation and a scaling of its columns. This is a trivial limitation common to all blind estimators. In this work, we have resolved it by assuming that the first transmitted symbol for each relay is known to the destination [14]. 